\title{
Industrialización de los crustáceos para la obtención de Quitosano en ungüento con efecto cicatrizante
}

${ }^{(1)}$ Teonila García Zapata ${ }^{(2)}$ Johana Melissa Roca Ortega

\begin{abstract}
RESUMEN
Entre el 20 a $30 \%$ del peso vivo de la especie de crustáceos es utilizado para la alimentación humana; el resto está constituido por visceras y exoesqueleto, especialmente de los caparazones de cangrejo peludo, cangrejo violáceo, jaiva, langosta, langostino que se encuentra en mayor cantidad a lo largo de las costas del litoral peruano, que son considerados como contaminantes ambientales (desechos), estos constituyen compuestos de valor comercial no aprovechados, que pueden ser utilizados para la obtención de dos biopolímeros especializados de alto valor agregado establecido a nivel mundial: la quitina y su derivado funcional, el quitosano, que permite obtener un producto natural con propiedades terapéuticas que ayude en el tratamiento de quemaduras. El objetivo de la presente investigación es desarrollar y presentar el producto obtenido en ungüento o crema para incursionar en competencia con los productos químicos de mayores costos, manteniendo las mismas propiedades; como la estimulación del crecimiento de nuevo tejido y contribuir a sanar las heridas.
\end{abstract}

Palabras clave: Quitina, quitosano, exoesqueleto de crustáceos, ungüento cicatrizante.

INDUSTRIALIZATION OF CRUSTACEANS FOR OBTAINING CHITOSAN IN EFFECT OINTMENT WOUND HEALING

\section{ABSTRACT}

Between 20 to 30, the weight of the kind of crust,Crustaceans is used human food, no human is the rest consisting of viceras and exoskeleton, especially the carapaces of hairy crab, crab raped her ceo, jaivas, lobster, shrimp that are found in greater numbers along the coasts of the Peruvian coast, which are regarded as environmental pollutants (waste) These compounds are commercially valuable untapped, which can be used to obtain two biopolymers specialized high valueadded established worldwide: chitin and its derivative functional, the chitosan, giving a natural product with therapeutic properties that help in the treatment of burns. The objective of this research is to develop and introduce the product in ointment or cream to go into competition with chemicals to higher costs, while maintaining the same properties, such as the stimulation of growth of new tissue and help heal the wounds.

Keywords: chitin, chitosan, exoskeleton of crustaceans, healing ointment.

\section{INTRODUCCIÓN}

La presente investigación pretende aportar a la industria farmacéutica un producto que haciendo uso de los desechos de los crustáceos se pueda obtener una forma farmacéutica semisólida, especialmente a partir de los caparazones del cangrejo, que al ser una alternativa natural, se compare en condiciones farmacoquímicas con las ya existentes en el mercado.

Durante el desarrollo de la investigación se ha tenido en consideración una serie de aspectos experimentales de consideraciones farmocotécnicas que conlleven a la elaboración de una forma farmacéutica semisólida tales como, la elección del principio activo adecuado para el tipo de afección. Elección de la forma farmacéutica y excipientes adecuados que incorporen y aseguren su estabilidad física y química, así como presentar una adecuada extensibilidad y adaptabilidad a la superficie y cavidades cutáneas y finalmente la permeabilidad de la piel y efectos cosméticos del vehículo para una adecuada absorción (dermis, epidermis o tejido celular subcutáneo). Aplicando conjuntamente las premisas anteriormente expuestas, y asumiendo que el medicamento está correctamente aplicado podrá obtenerse el éxito terapéutico del mismo.

Asimismo se podrá apreciar los diferentes ejemplares de formas farmacéuticas de tipo tópico que se clasifican de acuerdo a la lesión a tratar, las de tipo crónico (heridas y costras) y las de tipo agudo (eritema, vesículas, ampollas y exudación).

\section{JUSTIFICACIÓN}

El consumo de los crustáceos deja como desechos, aproximadamente, entre el 70 y $80 \%$, considerados contaminantes y están constituidos por sus vísceras y exoesqueleto. Al hacer uso de estos desechos, la presente investigación estaría aportando con el control de la contaminación ambiental y generando valor agregado al producir un ungüento natural.

Los desechos pueden aprovecharse para la obtención de dos biopolímeros especializados de alto valor agregado establecido a nivel mundial: la quitina y su derivado funcional, el quitosano, generando de esta manera mayor valor comercial y aportando con la medicina natural en fuerte competencia con los productos químicos importados.

1 Magíster en Promoción del Desarrollo, Docente principal de la Facultad de Ingeniería Industrial, Departamento Académico de Producción y Gestión Industrial teogaza57@yahoo.es

2 Químico Farmacéutica, estudiante de la maestría en ingeniería Industrial de la FII de la UNMSM. johanarocao@hotmail.com 
Los derivados de quitina tienen un inmenso campo de aplicación con relevante valor económico, por ejemplo en la utilización en la medicina por sus propiedades antimicrobianas y capacidad de retención de humedad. En la industria textil, para evitar el encogimiento de los tejidos, fija el color de componentes de fibras que se utilizan en la mejora de lanas, impermeabilización de algodones y linos.

En el tratamiento de agua, para removedores de iones metálicos, quelantes de metales de transición y contaminantes ambientales, floculantes, coagulantes y precipitantes de proteínas, aminoácidos, tintes, colorantes, algas, aceites, metales radioactivos, partículas en suspensión y pesticidas.

En la industria alimentaria para aditivos en los alimentos, espesantes, gelificantes y emulsionantes, agentes que previene la precipitación del vinagre, aditivos con características nutricionales, aditivos para la alimentación animal, envoltura y recubrimiento protector de alimentos, retrasa el envejecimiento, disminuye la oxidación, disminuye las perdidas por transpiración y protege frente al ataque de hongos, entre otros usos.

Por lo tanto la presente investigación aportará en lo económico, social y medio ambiental del país; asimismo, generará mayores puestos de trabajo formando una cadena productiva con la pesca, la industria farmacéutica, la preservación del medio ambiente y los consumidores finales.

\section{OBJETIVOS DE LA INVESTIGACIÓN}

\section{Objetivo General}

Obtener, diseñar y caracterizar, una forma farmacéutica semisólida (ungüento) a partir de la quitina de los crustáceos que sea de aplicación tópica y nos brinde efecto cicatrizante.

\section{Objetivos Específicos}

1. Extraer y aislar la quitina.

2. Obtener quitosano a partir de la quitina.

3. Caracterizar la quitina y el quitosano.

4. Comprobar el efecto cicatrizante del quitosano bajo la forma farmacéutica de ungüento.

\section{MARCO TEÓRICO}

\section{Obtención del quitosano mediante el Método Químico y Método enzimático}

La obtención del quitosano se produce por desacetilación de la quitina y se puede realizar mediante pro- cesos químicos o enzimáticos. Sin embargo, las condiciones específicas de la reacción dependerán de diversos factores, tales como el material de partida, el tratamiento previo, y el grado de desacetilación deseado.

Método Químico: se puede llevar a cabo de dos formas, homogénea y heterogénea.

a) La desacetilación homogénea. MIMA S, MIYA M, IWAMOTO R. y YOSHIKAWA S. (1983) explican que esta consiste en que la quitina es suspendida en el álcali y la suspensión es refrigerada con hielo para disolver la quitina en la solución. Luego se somete a desacetilación a temperaturas cercanas a la del ambiente durante períodos largos de tiempo. Esto permite que la reacción no se localice en determinados lugares de la cadena y que el ataque a los grupos amidas sea más uniforme.

b) La desacetilación heterogénea. Consiste en que las moléculas de quitina son dispersadas en una solución alcalina caliente, generalmente de hidróxido de sodio. Las condiciones en las que se lleva a cabo la desacetilación heterogénea pueden reducir la longitud de la cadena, por este motivo es conveniente repetir varias veces el tratamiento alcalino por cortos periodos de tiempo y aislando el producto en cada etapa. Para disminuir la pérdida de peso molecular del polímero es conveniente la ausencia de oxígeno o la presencia de un antioxidante para evitar su despolimerización.

Se ha demostrado que mientras que el quitosano obtenido en el proceso heterogéneo presenta polidispersión del grado de acetilación de sus cadenas, mientras que el obtenido por vía homogénea tienen la misma composición.

\section{Método Enzimático:}

GOYCOCHEA F. (1998) nos habla que la ventaja de este método respecto al químico es la obtención de un material uniforme en sus propiedades físicas y químicas, hecho muy apreciado para aplicaciones biomédicas. La quitina desacetilasa es la enzima que cataliza la conversión de quitina a quitosano por la desacetilación de los residuos N-acetil-D-glucosamina. La limitación de este método es que la enzima no es muy efectiva en la desacetilación de quitina insoluble, y por lo tanto es necesario un pre tratamiento.

En la actualidad, se exploran otros métodos más novedosos para desacetilar la quitina que hace uso 
de la radiación con microondas o de tratamientos termo-mecánicos, entre otros.

Caracterizacion del Quitosano: Método espectrofotométrico (IR), solubilidad y determinación del grado de desacetilación

ROBERTS G. (1998) explica que tanto la composición de las cadenas de quitosano, como sus dimensiones, suelen variar dependiendo del material de partida y de la rigurosidad del método de obtención.

Por este motivo, la aplicación de método espectrofotométrico (IR), la solubilidad y el grado de desacetilación son parámetros que se deben conocer para caracterizar una muestra de este polisacárido, ya que tienen gran incidencia en sus propiedades. Otros parámetros a determinar para su caracterización más completa serían, el peso molecular, el porcentaje de humedad, el contenido de cenizas, las proteínas totales, la cristalinidad, la determinación del contenido de material insoluble, etc.

\section{MÉTODO ESPECTOFOTOMÉTRICO (IR)}

VILCHEZ S. (2005) señala que uno de los métodos mas usados para la identificación de quitina y quitosano es la identificación por Espectrofotometría Infrarroja (IR). En el análisis de estos compuestos por espectroscopia IR se puede identificar, principalmente, los grupos funcionales amida y carbonilo. Para la quitina las vibraciones de estiramiento del grupo $\mathrm{NH}$ tienen dos frecuencias moderadamente intensas. En los espectros de muestra sólida, estas bandas se observan en rango de 3500 a $3180 \mathrm{~cm} " 1$ debido al enlazamiento de hidrógeno; la frecuencia del carbonilo se observa en la banda intensa en la región de 1680 a $1630 \mathrm{~cm}$ "1 y una banda intensa en la región de 1640 a 1550 que corresponde a la deformación del enlace $\mathrm{NJH}$, cuando se examina en estado sólido. Mientras que para el quitosano las bandas de intensidad media correspondientes a vibraciones de estiramiento del $\mathrm{NH}$ ubicadas en la región de $3500-3300$ cm' y la banda de absorción de intensidad media a fuertes relativa a vibraciones de deformación del grupo amino a una frecuencia entre 1640 a 1500 cn T1.

\section{LA SOLUBILIDAD}

La presencia de grupos amino a lo largo de la cadena de quitosano permite la disolución de esta macromolécula en disoluciones de ácidos diluidos, por medio de la protonación de esos grupos. Al adquirir carga positiva la amina, el quitosano aumenta su capacidad hidrofílica y pasa a ser soluble en soluciones acidas diluidas formando sales ya que el pKa del grupo amino en ei quitosano es 6,5. El quitosano se puede solubilizar en ácido clorhídrico, bromhídrico, iodhídrico, nítrico y perclórico diluidos. En cambio, es insoluble en ácido sulfúrico diluido.

También es insoluble en la gran mayoría de disolventes orgánicos como el alcohol. El quitosano, al igual que la quitina, es insoluble en agua.

\section{DETERMINACIÓN DEL GRADO DE DESACETILACIÓN}

El grado de desacetilación se define como el contenido en grupos aminos presentes en la cadena polimérica. Existen numerosos métodos para determinar el grado de desacetilación del quitosano basados en diversas técnicas. Entre estas técnicas destacan la espectroscopia de infrarrojo (IR), la espectroscopia de UV, La espectroscopia de RMN, la potenciometría y la conductimetría .

\section{Valoración potenciométrica}

Consiste en disolver quitosano en un exceso conocido de ácido clorhídrico y se valora con hidróxido de sodio. Se obtiene, así, una curva de $\mathrm{pH}$ frente a volumen de $\mathrm{NaOH}$ añadido que presenta dos puntos de inflexión. La diferencia de volumen entre estos dos puntos se corresponde con el ácido consumido para la protonación de los grupos amino y permite determinar el grado de desacetilación. La valoración se realiza utilizando un potenciómetro.

\section{APLICACIONES DEL QUITOSANO EN MEDICINA Y FARMACIA}

PENICHE C. (2006) expresa que el quitosano, debido a sus propiedades físico-químicas, funcionales y biológicas, tiene una gran variedad de aplicaciones que abarcan campos tan diferentes como la medicina, la farmacia, la agricultura, la alimentación y el sector textil entre otros.

En la tabla N.$^{\circ} 1$ que se muestra a continuación se observa la aplicación del quitosano en las diferentes áreas.

El quitosano es un polímero biocompatible, biodegradable, no-tóxico y muco adhesivo, lo que la hace atractiva para aplicaciones en medicina y farmacia. Es degradada por la lizosima y la lipasa. Los productos de la degradación enzimática del quitosano no son tóxicos. Además, el quitosano es un buen hemostático. Se sabe que el quitosano es hipocolesterolémico e hipolipidémico, posee actividad antimicrobiana, antiviral y antitumoral. 
TABLA N. 1: Aplicación del quitosano en diferentes áreas

\begin{tabular}{|c|c|}
\hline ÁREAS & APLICACIONES \\
\hline $\begin{array}{l}\text { Tratamientos } \\
\text { de agua }\end{array}$ & $\begin{array}{l}\text { Removedores de iones metálicos. } \\
\text { Quelantes de metales de transición y contaminantes ambientales. } \\
\text { Floculantes, coagulantes y precipitantes de proteínas, } \\
\text { aminoácidos, tintes, colorantes, algas, aceites, metales radioactivos, } \\
\text { partículas en suspensión y pesticidas. }\end{array}$ \\
\hline $\begin{array}{l}\text { Industria } \\
\text { Alimentaria }\end{array}$ & $\begin{array}{l}\text { Aditivos en los alimentos } \\
\text { - Espesantes, gelificantes y emulsionantes. } \\
\text { - Mejora la textura. } \\
\text { - Estabilizantes del color. } \\
\text { - Aditivos con características nutricionales. } \\
\text { - Aditivos para la alimentación animal. } \\
\text { - Envoltura y recubrimiento protector de alimentos } \\
\text { - Retrasa el envejecimiento. } \\
\text { - Disminuye la oxidación. } \\
\text { - Disminuye las perdidas por transpiración y } \\
\text { - Protege frente al ataque de hongos. }\end{array}$ \\
\hline $\begin{array}{l}\text { Procesos } \\
\text { industriales }\end{array}$ & $\begin{array}{l}\text { Agente purificador de azúcar. } \\
\text { Clarificador en industrias de bebidas. } \\
\text { Coagulación del queso. } \\
\text { Retardador del oscurecimiento enzimático de manzana y pera. }\end{array}$ \\
\hline Medicina & $\begin{array}{l}\text { Propiedades antimicrobianas. } \\
\text { Capacidad de retención de humedad. }\end{array}$ \\
\hline Biotecnología & $\begin{array}{l}\text { * Inmovilización de enzimas. } \\
\text { Separación de proteínas. } \\
\text { Inmovilización celular. } \\
\text { Reacción con aldehídos. } \\
\text { Captación de células y enzimas. }\end{array}$ \\
\hline Agricultura & $\begin{array}{l}\text { Bioestimulante de plantas en tratamientos de semillas, raíces y hojas. } \\
\text { En tratamientos post cosecha de frutas y verduras con el fin de aumentar su } \\
\text { conservación. } \\
\text { Recubrimientos de semilla. } \\
\text { - Conservación de frutas. } \\
\text { Protección frente a plagas y hongos, virucida. } \\
\text { Estimulante del crecimiento. }\end{array}$ \\
\hline Cosméticos & $\begin{array}{l}\text { Propiedades humectantes. } \\
\text { Propiedades abrasivas. }\end{array}$ \\
\hline $\begin{array}{l}\text { Industria } \\
\text { Papelera }\end{array}$ & $\begin{array}{l}\text { Elaboración de papeles. } \\
\text { Aumenta el rendimiento de la pulpa. } \\
\text { La capacidad de retención de agua. }\end{array}$ \\
\hline $\begin{array}{l}\text { Tecnologías de } \\
\text { membrana }\end{array}$ & $\begin{array}{l}\text { Para la separación de componentes. } \\
\text { Absorbentes de encapsulación. } \\
\text { Control de permeabilidad. } \\
\text { Osmosis inversa. }\end{array}$ \\
\hline $\begin{array}{l}\text { Industria } \\
\text { Textil }\end{array}$ & $\begin{array}{l}\text { Evita el encogimiento de los tejidos. } \\
\text { Fija el color. } \\
\text { Componentes de fibras que se utilizan en la mejora de lanas. } \\
\text { Impermeabilización de algodones y linos. }\end{array}$ \\
\hline $\begin{array}{l}\text { Industria } \\
\text { Farmacéutica }\end{array}$ & * Liberación controlada de sustancias. \\
\hline
\end{tabular}

FUENTE: PENICHE C. Estudios sobre quitina y quitosana. Universidad de La Habana; 2006. 
El quitosano presenta actividad cicatrizante, y se ha sugerido que el mecanismo por el cual él ejerce esta acción es mediante la activación de neutrófilos y macrófagos, la migración de polimorfo nuclear y células mononucleares, acelerando la regeneración de tejido conectivo y angiogénesis. Es posible que las moléculas positivamente cargadas de quitosano adsorban algunas sustancias implicadas en la proliferación de célula y la migración, como factores de crecimiento y citocinas, del plasma en la sangre o exudado en la herida y que las sustancias adsorbidas estimulan la proliferación de célula y la migración.

\section{CICATRIZACIÓN DE LAS HERIDAS, FASES y TIPOS}

La cicatrización es el conjunto de procesos biológicos, físico-químicos y celulares que se producen como respuesta de los tejidos a una lesión y tiene como finalidad, obtener la recuperación funcional de los mismos, mediante la formación de un tejido fibroso.

Independientemente del tipo de herida de que se trate y de la extensión que abarque la pérdida de tejido, cualquier curación de herida discurren en fases que se solapan en el tiempo y no pueden ser disociadas unas de otras, las cuales son:

a) Fase inflamatoria/exudativa: Hemostasia y limpieza de las heridas. La fase inflamatoria / exudativa se inicia en el momento en que se produce la herida y su duración es, aproximadamente, de tres días, dependiendo de las condiciones fisiológicas. Las primeras reacciones vasculares y celulares consisten en la coagulación y la hemostasia, y concluyen después de haber transcurrido, aproximadamente, 10 minutos. Por medio de la dilatación vascular y un aumento de la permeabilidad vascular se consigue intensificar la exudación de plasma sanguíneo en el intersticio. Con ello se fomenta la migración de los leucocitos hacia la zona de la herida, sobre todo de granulocitos, y macrófagos neutrófilos, cuya función prioritaria consiste en limpiar y proteger a la herida de posibles infecciones a través de la fagocitosis. Al mismo tiempo, liberan mediadores bioquímicamente activos, que activan y estimulan células de gran importancia para la siguiente fase del proceso curativo de la herida. Los macrófagos juegan un papel clave en esta fase.

b) Fase proliferativa o de proliferación de reconstitución de los tejidos granulares. En la segunda fase de la curación de la herida predomina la proliferación celular con el fin de alcanzar la reconstitución vascular y de volver a rellenar la zona defectuosa mediante el tejido granular. Esta fase comienza, aproximadamente, a partir del cuarto día desde que se produjo la herida, las condiciones necesarias ya han sido previamente establecidas en la fase inflamatoria-exudativa: los fibroblastos ilesos de los tejidos colindantes pueden migrar al coágulo y a la retícula de fibrina que han sido formados mediante la coagulación sanguínea y utilizarla como matriz provisoria, las citocinas, y los factores de crecimiento estimulan y regulan la migración y proliferación de las células encargadas de la reconstitución de tejidos y vasos.

c) Fase de diferenciación y de reconstitución: Maduración, cicatrización y epitelización. Aproximadamente entre el $6^{\circ}$ y el $10^{\circ}$ día comienza la maduración de las fibras de colágeno. La herida se contrae, se reduce cada vez más la presencia vascular y de agua en el tejido granular, que gana en consistencia y se transforma finalmente en el tejido cicatricial. La epitelización cierra el proceso de curación de la herida. Este proceso incluye la reconstitución de las células epidemiales a través de la mitosis y la migración celular, principalmente desde los bordes de la herida. Los diferentes tipos de cicatrización se pueden observar el en la tabla N. ${ }^{\circ} 2$.

Modelos de formas farmacéuticas de tipo tópico Se clasifican de acuerdo a la lesión a tratar:

a) Tratamiento de lesiones de tipo crónico. Entre estas lesiones se tienen: heridas y costras, xerosis, liquenrificación, y descamación. Las formas farmacéuticas de este grupo deben presentar como característica principal la oclu-

TABLA N.․ 2: TIPOS DE CICATRIZACIÓN

\begin{tabular}{|l|l|}
\hline \multicolumn{1}{|c|}{ Cicatrización por primera intención } & \multicolumn{1}{c|}{ Cicatrización por segunda intención } \\
\hline Poca pérdida de tejido & Bordes superpuestos \\
\hline Tejidos de granulación en pequeña cantidad & Cicatriz pequeña \\
\hline Evolución rápida & Gran pérdida de tejido \\
\hline Bordes tortuosos & Tejidos de granulación en gran cantidad \\
\hline Cicatriz grande & Evolución lenta \\
\hline
\end{tabular}

FUENTE: PENICHE C. Estudios sobre quitina y quitosana Universidad de La Habana; 2006 
sividad, que conlleva a evitar la pérdida de agua y genera un efecto hidratante acusado.

Como ejemplos de estas formas farmacéuticas se señalan las siguientes: apositos oclusivos, pomadas propiamente dichas, ungüentos hidrófobos o lipogeles, pomadas absorbentes de agua, pomadas hidrofílicas, cremas A/O.

b) Entre las lesiones o consecuencias de las lesiones se tiene: eritema, vesículas, ampollas y exudación. Las formas farmacéuticas de este grupo deben presentar como característica principal un mayor efecto penetrante, que conlleva a la evaporación del agua y rebajar la hidratación. Como ejemplo de estas formas farmacéuticas tenemos: hidrogeles, polvos, pastas acuosas, lociones, suspensiones, soluciones, cremas O/A.

\section{ESTUDIO QUÍMICO DEL QUITOSANO Obtención de la quitina}

Son cinco las etapas para la obtención de la quitina:

1. Recolección. Los Cangrejos de la variedad "peludo" Cáncer cetosus se adquirirán en el terminal pesquero de Ventanilla en el Callao.

2. Acondicionamiento. Los cangrejos serán separados de sus caparazones, para luego ser lavados de manera acuciosa con abundante agua, separando los restos orgánicos que pudieran estar presentes. Posteriormente, se desinfectarán con una solución de hipoclorito de sodio al $0.5 \%$, para luego ser lavados con agua $y$, finalmente, llevados a una estufa por 48 horas a $50^{\circ} \mathrm{C}$ hasta peso constante. Los caparazones secos se someterán a reducción de tamaño de partícula, con ayuda de un molino manual buscando obtener, aproximadamente, una cantidad equivalente a $400 \mathrm{~g}(409.81 \mathrm{~g})$ de material en polvo que será guardado en un recipiente de vidrio color ámbar cerrado herméticamente.

3. Desmineralización. Pesar 200 gramos de polvo del crustáceo y colocarlo en un matraz de 10 litros que contiene 6 litros de ácido clorhídrico 2 $\mathrm{N}$, la adición debe realizarse de manera lenta para evitar el levantamiento de la muestra por efervescencia, y dejar en reposo por un tiempo de 24 horas, decantar y proceder al lavado de la muestra con abundante agua hasta neutralidad para luego ser colocado en una estufa a una temperatura de $50{ }^{\circ} \mathrm{C}$ por 12 horas.

4. Desproteinización. La muestra es tratada con 2 Litros de hidróxido de sodio $2 \mathrm{~N}$ primero a temperatura ambiente por 24 horas y luego a baño maría a $60{ }^{\circ} \mathrm{C}$, por un período de 4 horas con agitación constante para asegurar la despro- teinización. Pasado el tiempo establecido, se lava la muestra con abundante agua hasta neutralidad para luego ser lavado con alcohol y finalmente secado en una estufa a $50{ }^{\circ} \mathrm{C}$ por 12 horas.

5. Decoloración. La muestra obtenida en el proceso anterior, se somete al proceso de decoloración. Para ello se adiciona $500 \mathrm{~mL}$ de agua destilada y se le llevó a baño maría por 15 minutos luego se le adiciona 500 mg de permanganato de potasio y se continúa con el calentamiento por 10 min más, para finalmente adicionarle $2 \mathrm{~g}$ de ácido oxálico con agitación constante hasta decoloración de la muestra; se deja enfriar y luego se lavado hasta neutralidad. La muestra finalmente se deseca en estufa con flujo de aire a $60{ }^{\circ} \mathrm{C}$ por 12 horas.

\section{OBTENCIÓN DEL QUITOSANO}

La quitina obtenida, se somete al proceso de desacetilación; para ello se pesan 22 gr de quitina, y se colocó en un equipo de reflujo con $500 \mathrm{~mL}$ de hidróxido de sodio al $50 \%$ y a una temperatura de $120{ }^{\circ} \mathrm{C}$ con agitación constante por un periodo de 3 días por intervalos de tiempo de 8 horas cada uno. Transcurrido el periodo, la muestra es lavada con abundante agua hasta neutralidad, y finalmente secada en estufa a una temperatura de $60{ }^{\circ} \mathrm{C}$ por 24 horas.

\section{Caracterización del quitosano}

Método espectrofotométrico (IR). La quitina y quitosano serán identificados mediante espectroscopia infrarroja (IR), utilizando un espectrofotómetro PERKIN ELMER FT-IR Modelo: Spectrum 1000. Los espectros obtenidos se interpretarán y compararán.

Solubilidad. Ensayos de Solubilidad: en 5 tubos de ensayo se coloca una pequeña porción de la muestra (100mg) y se agrega $5 \mathrm{~mL}$ del solvente respectivo: Agua, etanol, ácido acético, ácido oxálico y ácido clorhídrico, se agita, se deja en reposo por 24 horas y se observan los resultados.

\section{Determinación del grado de desacetilación (dga)}

Método. Valoración titulométrica de los grupos aminos.

Procedimiento. Para mediciones precisas, es necesario trabajar con un potenciómetro calibrado. Para la determinación del contenido de grupos amino de la muestra de quitosano, se procede a la disolución de $0,1 \mathrm{~g}$ de quitosano en $20 \mathrm{~mL}$ de ácido 
clorhídrico $0,3 \mathrm{M}$, luego se titula con una solución de hidróxido de sodio $0.1 \mathrm{M}$, la cual es valorada previamente con biftalato de potasio como patrón.

La valoración se lleva a cabo midiendo el cambio de $\mathrm{pH}$ cada $2 \mathrm{~mL}$ de base añadida, la adición se realiza de forma lenta y con agitación continua para homogenizar la solución y evitar errores debido a la posible precipitación del polímero. Las mediciones se realizan 3 veces por muestra. La diferencia entre los dos puntos de inflexión en la curva de titulación co-rresponde a la cantidad de ácido requerido para protonar los grupos aminos del quitosano, la concentración de estos se determina utilizando la expresión:

$$
\% \mathrm{NH} 2=\frac{16.1(\mathrm{Y}-\mathrm{X}) \mathrm{xF}}{\mathrm{W}}
$$

Donde:

"Y" = Punto de inflexión mayor (expresado como volumen)

"X" = Punto de inflexión menor (expresado como volumen)

"F" = Molaridad de la solución de $\mathrm{NaOH}$

"W" = Peso en gramos de la muestra

$16.1=$ Valor relacionado con el peso equivalente del quitosano

\section{DISEÑO DE LA FORMA FARMACÉUTICA (UNGÜENTO) DE APLICACIÓN TÓPICA A BASE DE QUITOSANO}

Objetivo. Desarrollar una forma farmacéutica tópica (ungüento) estable a base de quitosano que conserve el efecto cicatrizante.

Diseño. Para el diseño, se toma como principal referencia que la forma farmacéutica a elaborar sería utilizada tópicamente como cicatrizante, por tal motivo esta deberá contar con poder de penetración a través de la piel, por lo que se pensó en una forma farmacéutica semisólida como un gel o ungüento.

Dadas las propiedades físico-químicas del quitosano obtenido y la solubilidad, se optó por diseñar un ungüento.

Los ungüentos son preparaciones semisólidas destinadas para la aplicación externa sobre la piel o las membranas mucosas. Las bases para ungüentos que se utilizan como vehículos se pueden clasificar en cuatro grupos generales:

- Bases de hidrocarburos (Vaselina blanca y el ungüento blanco)
* Bases de absorción (Vaselina hidrofílica y lanolina)

* Bases que se eliminan con agua (Ungüento hidrofílico)

* Bases hidrosolubles. (Ungüento de polietilenglicol)

La elección de la base para ungüentos depende de los factores:

* La acción deseada

* La naturaleza del medicamento

* La biodisponibilidad y estabilidad

* La vida útil requerida para el producto terminado

Dadas las propiedades físico-químicas del quitosano obtenido y la solubilidad, se opta por diseñar un ungüento con una base hidrocarbonada, la cual está representada por la vaselina blanca y el ungüento blanco, la que permite incorporar una cantidad mínima de componente acuoso. Sirven para mantener los medicamentos en contacto prolongado con la piel y actúan como vendaje oclusivo Las bases hidrocarbonadas se usan, principalmente, por sus efectos emolientes y son difíciles de eliminar. No se "secan" ni se modifican en forma notoria con el envejecimiento. Son muy recomendables en el proceso de cicatrización de heridas.

\section{PREPARACIÓN DEL UNGÜENTO}

Para la preparación del ungüento se seguirán los siguientes pasos:

1. Preparar una solución de quitosano disuelto en ácido acético al $1 \%$.

2. Neutralizar la solución de quitosano con gotas de hidróxido de sodio $1 \mathrm{~N}$, homogenizando hasta obtener un $\mathrm{pH}$ cercano al de la piel $(\mathrm{pH}=6.5)$.

3. Fundir la vaselina sólida a una temperatura de aproximadamente $60^{\circ} \mathrm{C}$ en un recipiente con agitación.

4. Incorporar la solución de quitosano, previamente neutralizado, al recipiente que contiene la vaselina fundida y se homogeniza.

5. Retirar el recipiente del calor y continuar agitando hasta que la mezcla llegue a temperatura ambiente.

6. Con este procedimiento se prepararán los siguientes ungüentos $0.25 \% \mathrm{P} / \mathrm{P}, 0.50 \% \mathrm{P} / \mathrm{P}, 1.00 \%$ $\mathrm{P} / \mathrm{P}$ y base del ungüento solo.

7. Una vez preparadas las diversas concentraciones de quitosano, se procederá a envasar los ungüentos en tubos colapsibles y potes de $100 \mathrm{~g}$, sanitizados con formol al $10 \%$.

Se reservan mayores detalles sobre la preparación por razones de secreto intelectual. 


\section{ESTUDIO FARMACOLÓGICO DEL QUITOSANO}

HOWES (1929) realizó estudios para la determinación de la fuerza de tensión, método que se aplicó para la determinación del efecto cicatrizante de los ungüentos a base de quitosano por el método de la fuerza de tensión (Método de Howes y col.).

Objetivo. Determinar el efecto cicatrizante del ungüento a base de quitosano en comparación con un control (Cicatrin $囚)$ por el método de la fuerza de tensión.

Grupos de estudio. Es un estudio experimental pre-clínico que se realiza en ratones. Cada grupo experimental contiene 8 animales según se muestra en la tabla N. ${ }^{\circ} 3$.

Alimentación. Dichos animales se mantuvieron durante todo el estudio experimental con alimentación y agua.

Fundamento. El método de la Fuerza de Tensión mide la fuerza necesaria para abrir una herida incisa de $1 \mathrm{~cm}$ de largo, realizada en el tercio anterior del lomo y perpendicular al eje longitudinal de un ratón.

\section{Metodología empleada para el experimento}

1. Ambientación o acondicionamiento. Los 48 ratones albinos hembras, CEP Balb/c/CNPB provenientes del Bioterio del Centro Nacional de Producción de Biológicos (INS), fueron distribuidos al azar en 6 grupos de 8 ratones cada grupo y colocados en jaulas individuales. Se mantuvieron en observación por un periodo de una semana (7 días), verificándose la condición óptima de los ratones para el estudio. A dichos animales se les mantuvo en un ambiente ventilado apropiadamente, en jaulas individuales, con alimento balanceado (INS) y agua, por un periodo de 7 días previos al inicio de la experiencia.
2. Depilación. Después de una semana de ambientarse los ratones en el lugar de trabajo, se procedió a depilarlos con crema depilatoria Veet $₫$ en el primer tercio dorsal anterior en un área, aproximada, de $2 \mathrm{~cm}^{2}$, se les aplicó anestesia con pentobarbital sódico por vía subcutánea a una concentración de $50 \mathrm{mg} / \mathrm{kg}$ de peso. La depilación se realizó previa humectación (con agua tibia) de la zona a depilar, luego se agregó la crema depilatoria Veet $\AA$ permaneciendo 3 minutos para ejercer su efecto. Finalmente, con la ayuda de gasas húmedas se retiró la crema. Posterior a la depilación, los ratones se colocan en sus respectivas jaulas individuales teniendo estos libre acceso a bebida y comida. La depilación se realiza 24 horas antes del procedimiento quirúrgico a fin de descartar cualquier reacción alérgica a la crema depilatoria.

3. Incisiones y sutura. Después de 24 horas de la depilación, se procedió a anestesiar a los ratones administrándoles una dosis de $50 \mathrm{mg} / \mathrm{Kg}$ de pentobarbital sódico vía subcutánea (tercio inferior del lomo). Se colocó al ratón sobre la mesa de trabajo, desinfectando el área depilada y marcando 2 puntos equidistantes en $1 \mathrm{~cm}$ y perpendicular al eje longitudinal del ratón (zona de corte). Se realizó el corte sobre la zona indicada, se unen los bordes con un punto de sutura con hilo esterilizado en la parte central del corte. Esta etapa se realiza cumpliendo condiciones de asepsia.

4. Aplicación del ungüento. Obtenido el punto de sutura, se administró en forma tópica la primera dosis del tratamiento sobre la incisión con la ayuda de un hisopo, para obtener una distribución homogénea sobre la incisión. Se aplicó tanto las formulaciones a base de quitosano a los controles, la base del ungüento y el Cicatrin ${ }^{\circledR}$ en sus respectivos grupos, mientras que el ratón blanco no recibió tratamiento. Se repitió el tratamiento cada 12 horas en un lapso de 72 horas.

TABLA N. ${ }^{\circ}$ 3: Estudio experimental preclínico

\begin{tabular}{|c|c|c|c|c|c|c|}
\hline \multirow{2}{*}{ MÉTODO } & \multicolumn{7}{|c|}{ Grupos experimentales } & \multirow{2}{*}{ Blanco } \\
\cline { 2 - 7 } & $1 \%$ & $0.50 \%$ & $0.25 \%$ & $\begin{array}{c}\text { Control del } \\
\text { Ungüento }\end{array}$ & Cicatrin \\
\hline TENSIOMÉTRICO & 6 & 6 & 6 & 6 & 6 & 6 \\
\hline HISTOLÓGICO & 2 & 2 & 2 & 2 & 2 & 2 \\
\hline TOTAL & 8 & 8 & 8 & 8 & 8 & 8 \\
\hline
\end{tabular}

FUENTE:: Elaboración propia. 
Determinación de la fuerza de tensión. Luego de 72 horas de tratamiento, se procedió a realizar la medición de la fuerza de tensión en el equipo de tensión. Para ello, los ratones son previamente anestesiados con una dosis de $100 \mathrm{mg} / \mathrm{kg}$ de pentobarbital sódico vía intraperitoneal. Luego se colocaron en la mesa de trabajo y se procedió a marcar los puntos donde se engancharan las agujas del equipo de tensión a 0,5 cm de ambos extremos del punto de sutura. Se retira con mucho cuidado el punto de sutura y se coloca al animal en posición decúbito ventral sobre el aparato de tensión, luego se insertan las agujas, se retira la base del recipiente y de inmediato se abre la bureta para dejar caer el agua hasta que se genera una fuerza de tensión capaz de abrir la herida en toda su longitud. Finalmente, se anota el nivel de agua requerido. Dando como resultado condiciones cicatrizantes óptimas con respecto al ratón blanco.

\section{CONCLUSIONES}

1. La caracterización de la quitina aislada de los caparazones de los crustáceos (Cangrejo peludo) cáncer cetosus, y el quitosano, obtenido por desacetilación química de la quitina, cumplen con las propiedades fisicoquímicas, de solubilidad, grado de desacetilación y espectroscopia IR.

2. Se comprobó que el quitosano bajo la forma farmacéutica semisólida (ungüento) presenta actividad terapéutica como cicatrizante externo.

3. Los estudios histológicos demuestran que los ungüentos a base de quitosano estimulan la proliferación y migración celular regenerando la dermis.

\section{RECOMENDACIONES}

1. Promover la transformación de desechos marinos en productos con actividad biológica.
2. Continuar los estudios clínicos de la actividad terapéutica como cicatrizante externo del ungüento a base de quitosano.

3. Continuar con el estudio y evaluación de la actividad cicatrizante de quitosano obtenido de otras especies marinas peruanas.

4. Promover los trabajos interdisciplinarios que para el caso se ha realizado entre ingeniería industrial y farmacia.

\section{REFERENCIAS BIBLIOGRÁFICAS}

1. Goycochea F. et al. Preparation of chitosan from squid (Loligo spp.) pen by a microwave accelerated thermochemical process. Advances in Chitin Science, Domard A, Roberts G, Várum K, Editors. Jacques André Publisher: Lyon. 1998: 78-83.

2. Howes E., Sooy J, Harvey S. The healing of wound as determined by their tensile strength 1929; 42.

3. Mima S., Miya M., Iwamoto R., Yoshikawa S. Highiy Deacetylated Chitosan and tts Properties. J Appl Polym Sci. 1983; 28(6):1909-1917.

4. Peniche C. Estudios sobre quitina y quitosana (Tesis para optar por el grado científico de doctor en ciencias).La Habana-Cuba. Universidad de La Habana; 2006: 153-170.

5. Roberts G. Chitosan production routes and their role in determining the structure and properties of the product. Advances in Chitin Science, Domard A, Roberts G, Várum K, Editors. Jacques André Publisher Lyon; 1998: 22-31.

6. Vílchez S. Nuevos tratamientos de lana con enzimas. Barcelona España. Universidad de Barcelona; 2005: 100-108. 